\title{
Review: probiotics are effective in preventing antibiotic-associated diarrhoea
}

D'Souza AL, Rajkumar C, Cooke J, et al. Probiotics in prevention of antibiotic associated diarrhoea: meta-analysis. BMJ 2002 Jun 8;324:1361-4.

\section{QUESTION: In patients being treated with antibiotics, does co-administration of probiotics reduce the incidence of diarrhoea?}

\section{Data sources}

Studies in any language (with English abstracts) were identified by searching Medline (1966-2000) with the terms probiotics, biotherapeutic agents, lactobacilli, antibiotic associated diarrhoea, and Clostridium difficile; the Cochrane Controlled Trials Register; and the Cochrane Database of Systematic Reviews.

\section{Study selection}

Studies were selected if they were randomised, doubleblind, placebo-controlled trials of probiotic treatment given in combination with antibiotics and diarrhoea prevention was reported.

\section{Data extraction}

Data were extracted on sample size; type, dose, and duration of probiotic treatment; and antibiotic studied. The outcome of interest was prevention of diarrhoea. Diarrhoea was defined as a change from the normal bowel habit with $\geq 2$ loose or watery stools for $\geq 2$ days.

\section{Main results}

9 trials (1214 patients) met the selection criteria. 2 of the trials studied children. No statistical heterogeneity or publication bias was detected among the 9 trials. The pooled odds ratio $(\mathrm{OR})$ showed that probiotic treatment was more effective than placebo in the prevention of diarrhea $(0.37,95 \%$ CI 0.26 to 0.53$) .4$ trials that used Saccharomyces boulardii (yeast trials) also favoured probiotic treatment (OR 0.39, CI 0.25 to 0.62 ) as did 5 that used lactobacilli or enterococci (non-yeast trials) (OR 0.34 , CI 0.19 to 0.61 )

\section{Conclusion}

In patients being treated with antibiotics, coadministration of probiotics reduces the incidence of diarrhoea.

\section{COMMENTARY}

The incidence of antibiotic-associated diarrhoea in hospitals ranges from 3.2\% to 29\%. ${ }^{1}$ Antibiotic-associated diarrhoea has been associated with an increased number of days of hospitalisation and higher medical costs. Probiotics are becoming increasingly available, and their lack of side effects makes them a particularly attractive option for preventing antibiotic-associated diarrhoea.

The well done review by D'Souza $e t$ al addresses whether coadministration of probiotics with antibiotics reduces the incidence of antibiotic-associated diarrhoea. Their analysis yields impressive ORs that show that probiotics seem to decrease the incidence of antibiotic-associated diarrhoea.

However, several points should be considered when interpreting the results. Firstly, clinical heterogeneity limits the potential generalisability of the results. The 9 studies had differences in probiotic agents administered, dosages, duration of administration of probiotic agents, and antibiotics. Secondly, the confidence intervals in this review may be too narrow because the authors used a fixed-effects model for analysis instead of the more conservative random-effects model; the latter may be more appropriate because statistical tests of heterogeneity in meta-analyses may have low statistical power. $^{2}$

Does this review provide strong enough evidence to integrate the use of probiotics into practice? My answer would be "no," mainly because of the clinical heterogeneity of the agents used thus far in published studies. The authors have appropriately written a conservative concluding statement in their review. I hope that future clinical trials will give a more definitive answer as probiotics undergo increasing scrutiny and standardisation. ${ }^{3}$ Future trials need to be characterised with the same scientific rigour that is applied to standard drugs.

Anthony D Harris, MD, MPH University of Maryland Baltimore Maryland

USA

1 McFarland LV. Diarrhea acquired in the hospital. Gastroenterol Clin North Am 1993;22:563-77.

Hardy RJ, Thompson SG. Detecting and describing heterogeneity in meta-analysis. Stat Med 1998;17:841-56.

Aeneity in meta-analysis. Stat Med 1998;1 7:841-56.

infectious diseases: a modern perspective on a traditional therapy. Clin Infect Dis 2001;32:1567-76.

Probiotics v placebo to prevent antibiotic-associated diarrhoea*

\begin{tabular}{|c|c|c|c|c|}
\hline \multirow[b]{2}{*}{ Outcome } & \multicolumn{2}{|c|}{ Weighted event rates } & \multirow[b]{2}{*}{$\mathrm{RBI}(95 \% \mathrm{Cl})$} & \multirow[b]{2}{*}{ NNT (CI) } \\
\hline & Probiotics & Placebo & & \\
\hline Proportion of patients without diarrhoea & $90 \%$ & $78 \%$ & $15 \%(9$ to 20$)$ & 9 (7 to 13$)$ \\
\hline
\end{tabular}

